\title{
An explicit integration finite element method for impact noise generation at squat
}

\author{
Z. Yang, Z. Li and R.P.B.J. Dollevoet \\ Section of Road and Railway Engineering, Faculty of Civil Engineering and Geosciences, \\ Delft University of Technology, \\ Stevinweg 1, $2628 \mathrm{CN}$, Delft, the Netherlands \\ Tel: +31 15 2781525, Fax: +31 15 2783443, E-mail: Z.Yang-1@tudelft.nl
}

\begin{abstract}
Summary
This paper presents a full finite element (FE) interaction model of wheel-track to study the wheel-rail impact noise caused by squat. The wheel, the rail and some other track components are modeled with finite elements in three dimensions, where necessary and appropriate. Realistic contact geometry, including geometric irregularity (squat) in the contact surfaces is considered. The integration is performed in the time domain with an explicit central difference scheme. For convergence, the Courant time step condition is enforced, which, together with the detailed modeling of the structural and continuum of the wheel-track system, effectively guarantees that vibration frequency of $10 \mathrm{kHz}$ or higher is reproduced. By making use of the calculated velocities and pressures on the vibrating surfaces, the boundary element method (BEM) based on Helmholtz equation is adopted to transform the vibration of the track into acoustic signal.
\end{abstract}

\section{Introduction}

Due to the requirements on improvement in passengers' comfort and in living environment standard, the noise problem of railways has attracted more and more attentions. The noise radiated by the railway system has significant influences on both passengers inside the trains and the residents along the railway lines. Many studies on railway noise have been carried out since the early 1970s and great progresses have been made during the past 40 years.

It has been widely accepted that, at conventional travelling speeds, the generation mechanism of the railway noise is mainly due to wheel and rail interaction [1]. According to different wheel-rail contact forms, the railway noise can be divided into three categories: rolling noise, impact noise and curve squeal noise. The knowledge about impact noise, due to its complex contact conditions over discontinuities of the rail-wheel surfaces and the possibility of loss of contact, lags far behind that of the other two. 
An early comprehensive study on impact noise was published by Vér et al. in 1976 [2], before which the studies focused on test and were largely qualitative. Vér et al. established an analytical model and defined geometric and dynamic variables which make contributions to impact noise generation by analysing the noise initiated at different types of discontinuities such as rail joints, frogs, switches, and wheel flats. Because of the limited modeling approaches and computing resources at that time, the analytical model was simple and failed to calculate the time history of impact force, let alone the spectral distribution of impact noise.

In reviewing the prior work, Remington [3] introduced the models and method of Newton and Clark for contact force calculation [4] into analysis of impact noise to tackle non-linear contact problem, pointing out a new direction for impact noise study. In addition, the geometric discontinuities of the rail and wheel surfaces were replaced with an average roughness spectrum in frequency domain as the excitation inputs for the calculation of impact vibration and noise.

With the development of numeric methods, finite element method was increasingly utilized to analysis the response of wheel-rail system. In the 1990s, Thompson et al. developed TWINS [1], which is regarded as the most advanced software so far for rolling noise calculation. A finite element model of the wheel was employed to predict its resonant frequencies and mode shapes. Since the linear frequency-domain contact model in TWINS was initially developed for solving rolling noise problem, the computationally efficient modal superposition method was employed to analysis the dynamic response of the system and the roughness spectrum could be directly applied as excitation. However, when it comes to the calculation of impact noise, the non-linear contact problem demands a time-domain approach. $\mathrm{Wu}$ and Thompson in [5] firstly employed a time-domain model to obtain the impact force between wheel and rail under non-linear contact conditions, and then, employing a linear approximation method, converted the impact force into force spectrum in frequency domain and further into an equivalent roughness spectrum also in frequency domain. On the basis of TWINS, the processed roughness spectrum was directly applied as inputs and the impact noise results could be obtained. This hybrid method managed to solve the problem of non-linearity, but the analysing frequency was limited to $5 \mathrm{kHz}$.

Instead of using simplified models of wheel and track, and the assumption of non-Hertzian contact $[5,6]$, this paper presents a new approach, with realistic wheel and track structure, geometry and materials, as well as accurate solution of impact-rolling contact with the continuum dynamics of the rail and wheel being taken into account, to predict the impact noise, of which the maximal frequency dealt with can be extended to $10 \mathrm{kHz}$.

Squat is a type of common local rail top deformation in the running band and mainly found on tangent sections and shallow curves of all types of tracks. On the Dutch railway network, hundreds of kilometers of track are infected with squats [7]. When a train runs over a severe squat, wheel-rail impact inevitably happens and simultaneously impact noise is generated and emitted. This process will be simulated in this paper. 


\section{Method}

\subsection{The numerical model}

As shown in fig. 1, a 3D finite element model was set up for the time domain simulation of a wheel rolling over a squat with the speed $\mathrm{v}$. A half of a wheelset was modeled with a section of railway track of 9.14 meters long. The wheel geometry corresponds to a passenger car wheel of the Dutch railway network with a radius of 0.46 meter; The rail is modeled as UIC60 with an inclination of 1:40. The squat position is $0.45 \mathrm{~m}$ away from the initial wheel position, and just over the edge of the sleeper where the support stiffness varies, which has been considered as an important contributing factor for squats development [7].

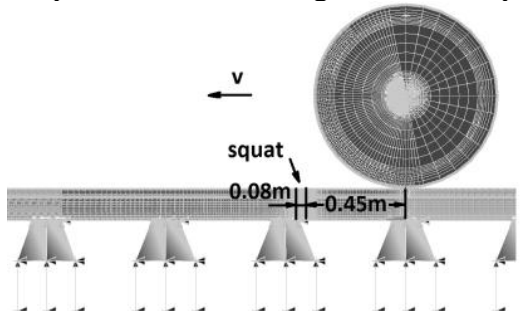

Fig.1. FE Wheel-Rail Interaction Model

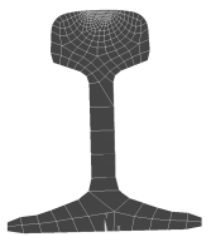

(a)Mesh 1

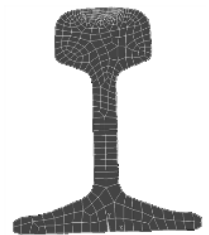

(b)Mesh 2

Fig.2. Meshes of the rail

The wheel and the rail were modeled with 8-node elements. In order to achieve a high accuracy of the solution with a reasonable model size, non-uniform meshing is used. Fig. 2 shows two meshing schemes applied to the cross-section of the rail. The amounts of elements of mesh 1 and mesh 2 are respectively 293 and 383. The results calculated by the two different meshes will be compared in the later analyses. The other track parameters involved in the model were taken from [8].

To apply a squat to the originally smooth surface of a rail for the simulation, geometry of a typical severe squat was measured with RAILPROF as [9]. Since the RAILPROF measures the longitudinal-vertical rail profile along the center line of the rail, while the maximum deviation of the vertical irregularity probably occurs off the center line, and cracks beneath the squat may affect the accuracy of the measurement, modification is made for a more realistic shape of squat in the model. Figure 3(a) shows the estimated shape of the squat applied to the model and figure 3(b) shows the surface of the rail with or without the squat.

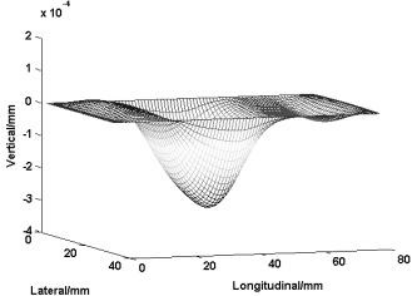

(a)Estimated squat shape

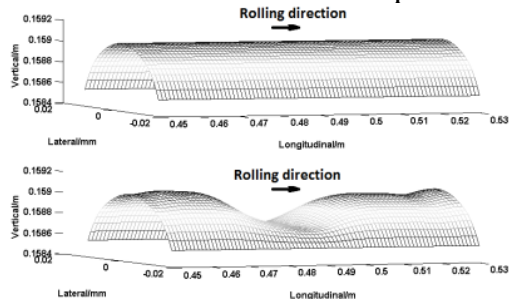

(b)Surfaces of the rail without(above) or with (below) a squat

Fig.3. Squat applied to the model 


\subsection{Noise radiation}

The Boundary element method is employed to transform the vibration of the wheel and rail into acoustic radiation [10]. In this case, a boundary surface enclosing a volume (rail or wheel) is surrounded by an ideal and homogeneous fluid medium (air). In the frequency domain, the acoustic wave propagation in an ideal fluid is governed by Helmholtz equation as:

$$
\nabla^{2} p+k^{2} p=0
$$

where $k=\omega / \mathrm{c}$, denotes the wave number; $\mathrm{c}$ is the sound velocity; $\omega=2 \pi f$ is the pulsation, and $p$ is the pressure at any point in the acoustic medium. Because of the non-linear character of impact problem, the transient track structural response is required to be computed in time domain. By applying the Fast Fourier Transform, velocities on the surface of the structure are converted into a frequency response, which can be taken as boundary conditions in eq. (2.2) for the BEM:

$$
\frac{\partial p}{\partial n}=-\mathrm{i} \rho \omega v_{n}
$$

Where $v_{\mathrm{n}}$ is the amplitude of the velocity of particle normal to the surface; $\rho$ is the density of the medium (air). By employing Green's theorem and boundary condition, the corresponding Helmholtz integral equation can be written as:

$C(\boldsymbol{r}) p(\boldsymbol{r})=-\int_{s_{y}}\left[i \rho \omega v_{n}\left(\boldsymbol{r}_{y}\right) G\left(\boldsymbol{r}, \boldsymbol{r}_{y}\right)-p\left(\boldsymbol{r}_{y}\right) \frac{\partial G\left(\boldsymbol{r}, \boldsymbol{r}_{y}\right)}{\partial n_{y}}\right] d S_{y}$

This equation allows the calculation of sound pressure at any receiver point of the acoustic domain. $G\left(\boldsymbol{r}, \boldsymbol{r}_{\boldsymbol{y}}\right)$ is the Green's function. $\boldsymbol{r}$ is the position vector of any field point; $\boldsymbol{r}_{y}$ is the position vector of a source point located at acoustic domain boundary and $\mathrm{C}$ is the jump term that depends on the location of $\boldsymbol{r}$. The BEM is employed to mesh the vibration surface and discretize the integral equation (2.3) into a linear equation system. Consequently, the radiated noise at any point in the field can be calculated.

\section{Results and analyses}

Relying on the FE Model and the noise calculation method mentioned above, the contact force, vibration and noise emission of the modeled wheel-rail system were obtained.

\subsection{Contact force}

Wheel-rail contact force is the fundamental excitation of the track structural vibration and the consequent noise emission. The contact forces calculated with mesh 1 with and without squat and with mesh 2 with squat are shown in fig. 4 . By comparing the contact forces between the cases with and without squat, it can be seen that the main frequency band at center frequencies of $3150 \mathrm{~Hz}$ is due to the intrinsic feature of track system rather than wheel-rail impact over squat. The 
other two main frequency bands at center frequency of $250 \mathrm{~Hz}$ and $1 \mathrm{kHz}$ agree well with the results in [5]. The mid-high frequency components $800 \mathrm{~Hz}-3 \mathrm{kHz}$ of contact force predicted by Mesh 2 is larger than that calculated by Mesh 1 model.

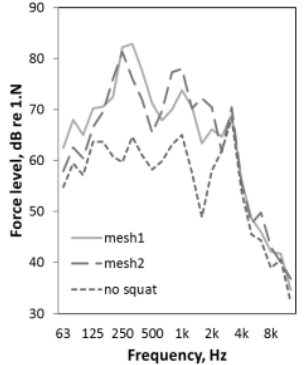

(a) Simulation results

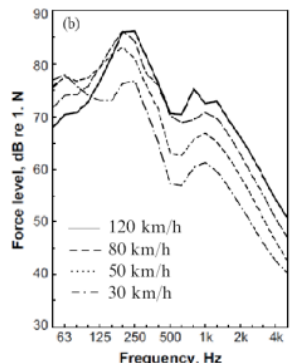

(b) Literature results[5]

Fig.4. One-third octave band spectra of contact force

In the wavelet power spectrum (WPS) in fig. 5, it is shown that the $1 \mathrm{kHz}$ frequency components mainly occur at the position of squat $(0.45-0.53 \mathrm{~m})$ and this phenomenon for contact force calculated by mesh 2 model is more obvious. It can be deduced that the mesh of rail affects less the position of the main frequency of the contact force but has stronger influence on the energy of the frequency. Dense mesh takes advantages of simulating high frequency components.
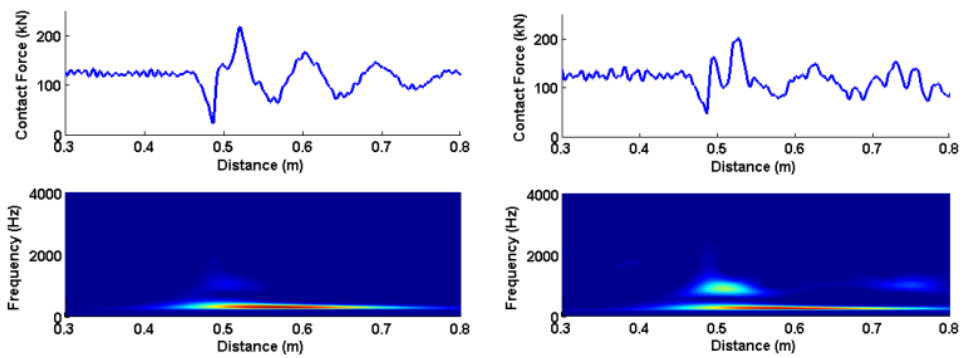

Fig.5. Time history and wavelet power spectra of contact force calculated by Mesh 1 (left) and Mesh 2 (right)

\subsection{Verification of the Vibration of wheel}

The calculated vibration accelerations of the wheel are verified with measured axle box accelerations in fig.6. The time-history vertical accelerations of the wheel simulated with Mesh 1 and Mesh 2 and the vertical accelerations at axle-boxes measured for twice at the same squat are compared in fig. 6 . In order to see the tendencies and differences of the simulated and measured results clearly, their $1 \mathrm{kHz}$ low-pass filtered signals are plotted below the original signals. As shown in fig. 6 (a), the simulating results have good agreements with the measured results in time domain, especially for the case of Mesh 2. In frequency domain, the one third octave band spectra in fig.6 (b) indicate that the wheel acceleration calculated by the Mesh 2 is in accordance with the measured results in the frequency range below $4 \mathrm{kHz}$; Two main frequency bands around $250 \mathrm{~Hz}$ and $1 \mathrm{kHz}$ 
are accurately calculated and the acceleration levels are more or less the same as the measurement. As to the model with Mesh 1, the two main frequencies of wheel accelerations are successfully predicted, but there exists a larger deviation in values of acceleration level.

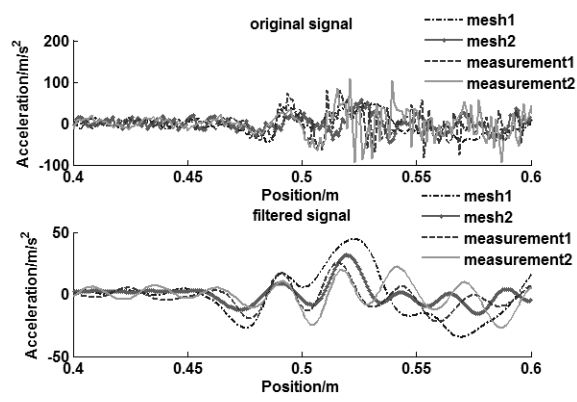

(a)Original (above) and filtered(below) time-histories

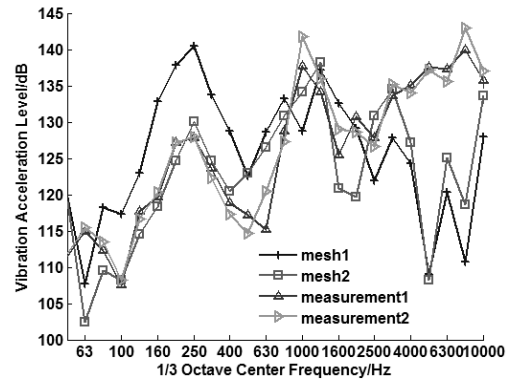

(b)One-third octave band spectra

Fig.6. Comparison of simulated and measured axle box acceleration

\subsection{Noise radiation}

According to the analyses of wheel-rail contact force and wheel vibration, a conclusion can be drawn that Mesh 2 is more accurate and suitable to solve high-frequency impact problem than Mesh 1 . Therefore, only the Mesh 2 model is applied to predict the noise generated by wheel-rail impact over the squat. The distribution of receivers employed to collect the acoustic signals generated by the track system is shown in fig. 7: Taking in-situ measurement scenario into consideration, the far-field receivers are placed at the same height as the wheel axle $(0.6 \mathrm{~m})$ to reduce the ground reflection influence and perpendicular to the wheel web plane with distances of $1.5 \mathrm{~m}, 3 \mathrm{~m}$ and $6 \mathrm{~m}$ respectively. The near-field receiver is located $0.1 \mathrm{~m}$ (approximate to $0 \mathrm{~m}$ ) beside the rail web.

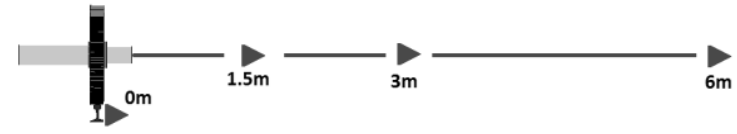

Fig. 7. Placement of sound receivers

On the basis of the BEM, the vibrating rail and wheel can be regarded as the acoustic emission source respectively. The acoustic results are calculated by the normal vibration velocities and pressures on the surface of the rail and the wheel to investigate the noise contribution by them. Because of the page limitation, only the near-field acoustic signal radiated by the rail is plotted in fig. 8 to present the wave shape of the simulated acoustic signals. The peak values of both time-history signal in fig. 8(a) and power spectrum in fig. 8(b) come out at the impact point. The sound pressure amplitude reaches up to $124 \mathrm{~Pa}$; The main frequencies of impact noise concentrate on $600 \mathrm{~Hz}$ and $1600-2500 \mathrm{~Hz}$ and its high frequency components can reach $6 \mathrm{kHz}$ or even higher . 

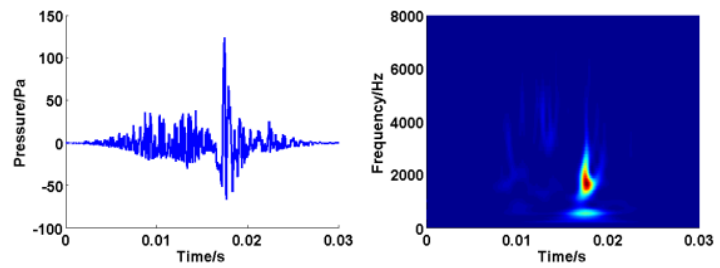

Fig. 8. Time-history (left) and WPS (right) of acoustic signal collected by receiver at $0 \mathrm{~m}$

One third octave of the acoustic signals collected by each receiver contributed by the rail and the wheel are shown in fig. 9(a) and 9(b) respectively. For the noise radiated by the rail, the main frequency bands of the near-field signal are around $500 \mathrm{~Hz}$ and $1600 \mathrm{~Hz}$, corresponding to the results in Fig. 8, and the main frequency bands of the far-field signals are around $315 \mathrm{~Hz}, 800 \mathrm{~Hz}$ and $1600 \mathrm{~Hz}$. As to the noise contributed by the wheel, the common main frequencies of both near-field signal and far-field signals are around $315 \mathrm{~Hz}$ and $3150 \mathrm{~Hz}$. The one-third octave curves of sound emitted by wheel also present 2 peaks at $6.3 \mathrm{kHz}$ and $10 \mathrm{kHz}$. Since the previous study on impact noise never extended the analyzing frequency range to more than $5 \mathrm{kHz}$, measurement is required for validation of these results.
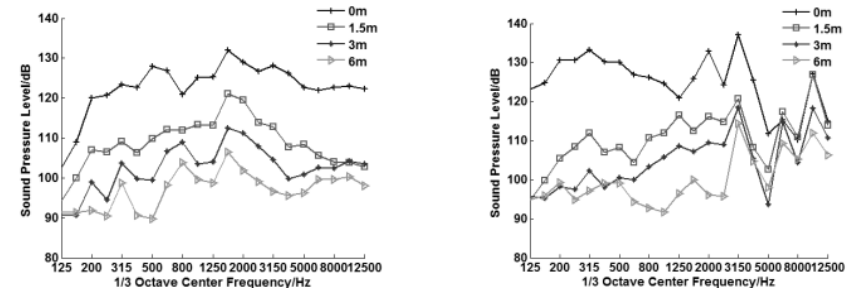

Fig. 9. One-third octave of impact noise radiated by the rail (left) and the wheel (right)

\section{Conclusion}

The wheel-rail contact force and the vibration of wheel at axle box simulated by the FE model were verified respectively by comparing with the literature and field measurements. The good agreements of the results qualify the model for simulating the noise generated by wheel-rail impact over the squat. The influence of rail mesh was investigated and it indicates that the results calculated by the model with reasonable denser mesh has a better agreement with test results.

The BEM based on Helmholtz equation was employed to transform the vibration simulated by the FE model into acoustic signal. The wavelet power spectra of acoustic signals show that, the frequency of impact noise can reach up to $6 \mathrm{kHz}$ and its main frequency ranges concentrate on $600 \mathrm{~Hz}$ and $1300-2500 \mathrm{~Hz}$. The one-third octave band curves indicates that the main frequencies of noise contributed by the rail and wheel are respectively $1500 \mathrm{~Hz}$ and $3150 \mathrm{~Hz}$ or even higher, which need to be proved by measurements. 


\section{Future work}

This is the first attempt to apply this explicit integration finite element method to simulate impact noise generation. Due to the track access restriction, field test fails to be performed. The vibration of the rail together with the impact noise propagation need to be measured in future to verify the simulation results. Taking the measurements as reference, mesh optimization of the model should be further studied. The refinement of wheel mesh is also necessary for accurate prediction. Weld and Insulated joints as more common discontinuity scenarios for impact noise generation will be modeled and their impact noise emissions will be studied on the base of this squat impact noise simulation in future work.

\section{References}

[1] Thompson, D.J. Jones, C.J.C.: A review of the modelling of wheel/rail noise generation, Journal of Sound and Vibration. 231(3), 519-536 (2000).

[2] Vér, I.L. Ventres, C.S. Myles, M.M.: Wheel/rail noise-part III: impact noise generation by wheel and rail discontinuities, Journal of Sound and Vibration. 46(3), 395-417 (1976).

[3] Remington, P.J.: Wheel/rail squeal and impact noise: What do we know? What don't we know? Where do we go from here?, Journal of Sound and Vibration. 116(2), 339-353 (1987).

[4] Newton, S.G. Clark, R.A.: An investigation into the dynamic effects on the track of wheelflats on railway vehicles, Journal of Mechanical Engineering Science. 21(4) 287-297 (1979).

[5] Wu, T.X. Thompson, D.J.: On the impact noise generation due to a wheel passing over rail joints, Journal of Sound and Vibration. 267(3) 485-496 (2003).

[6] Pieringer, A. Kropp, W. Thompson, D.J.: Investigation of the dynamic contact filter effect in vertical wheel/rail interaction using a $2 \mathrm{D}$ and a $3 \mathrm{D}$ non-Hertzian contact model, Wear. 271(1-2) 328-338 (2011).

[7] Li, Z. Zhao, X. Dollevoet, R.P.B.J. Molodova, M.: Differential wear and plastic deformation as causes of squat at track local stiffness change combined with other track short defects, Vehicle System Dynamics. 46 237-246 (2008).

[8] Zhao, X. Li, Z. Liu, J.: Wheel-rail impact and the dynamic forces at discrete supports of rails in the presence of singular rail surface defects, Rail and Rapid Transit. 226 124-139 (2011).

[9] Molodova, M. Li, Z. Dollevoet, R.P.B.J.: Axle box acceleration: Measurement and simulation for detection of short track defects, Wear. 271(1-2) 349-356 (2011).

[10] Wu, T.W.: Boundary Element Acoustics: Fundamentals and computer codes. Advances in boundary elements, Southampton, Boston, WIT press, 2000.

[11] Thompson, D.J.: Railway Noise and Vibration : Mechanisms, Modelling and Means of Control. Elsevier, 2009. 